\title{
Solution Methods for Thermo-elastic-Plastic FEM and \\ Its Application to Centrifugal Casting Mold*
}

\author{
By Yoshio EBISU**
}

\begin{abstract}
Synopsis
Three solution methods for thermo-elastic-plastic finite element analysis were studied with special emphases paid to the treatment of elastic-plastic transition. These are the iterative convergent method using "effective stiffness matrix" and two modified $r_{\mathrm{m} \text { in }}$ methods I and II. The computational results obtained were compared with each other in terms of the "followability" of the equivalent stress and the flow stress of the material.

A simple and efficient algorithm to automatically determine time increment was also presented. It was shown that when this algorithm is combined with the modified $r_{\mathrm{m} \text { in }}$ method II highly accurate solution can be obtained with less thermal load increments.

Computations were performed on the basis of the modified $r_{\mathrm{m} \text { in }}$ method II on centrifugal casting molds to analyze the influences of casting parameters on the stress-strain state of the molds.

The results revealed the importance of the adiabatic effect of refractory coating to enhance the thermal fatigue resistance of the bore surface of mold. The influences of the pouring temperature, the mold wall-thickness and the preheating temperature of mold were also clarified.

Mold warping or bowing was also discussed in conjunction with the "plastic work over a mold", which was found to be decreased considerably by increasing the thermal resistance of coating, by lowering the pouring temperature and by thickening the mold wall.
\end{abstract}

\section{Introduction}

The theories and formulations of nonlinear elasticplastic analysis by the use of finite element method (FEM) are now available in standard text books. ${ }^{1-4)}$ And the main concern at present seems to lie in the knowledge and understanding of constitutive relations of materials from the view point of materials scientists and in the solution methods for both material and geometric nonlinear behaviors. Besides, in regarding thermo-elastic-plastic and/or creep analysis, the problems become more complicated primarily because the temperature dependencies of material properties have to be taken into account.

Chapter II of this paper investigates the accuracy and stability of the solutions for thermoelastoplasticity problems with special attention paid to the treatment of elastic-plastic transition.

In connection with the treatment of the elasticplastic transitional elements, there are two categories in general: One is the method of yielding plural number of elements simultaneously for prescribed load increments, and the other is the method of adjusting load increments in such a way as to yield the elements one by one. The representative methods of the former are " mean stiffness method" of Marcal et $a l .{ }^{5,6)}$ and "initial stress method" of Zienkiewicz et al..$^{7,8)}$ The former also includes the method of Basombrío and Sarmiento ${ }^{9)}$ in which the stress increments after yielding are corrected to stay on the stress-strain curve of the material and a rather straightforward treatment by Snyder and Bathe ${ }^{10)}$ in which the stress increments during the transition are simply divided into elastic and elastic-plastic parts. The latter, initiated by Yamada et al. ${ }^{11)}$ and later known as the $r_{\min }$ method, includes its modified version by Inoue et al. ${ }^{12)}$ that is applied to thermal stress analysis.

In addition, there is a method presented by $\mathrm{Hsu}$ and $\mathrm{Too}^{13)}$ in which a stress-strain relation of material is approximated by a continuous function, thereby circumventing the above-mentioned problems of matching loading and stress-strain relation of the materials.

From the above points of view, the author investigated three solution methods: Method I which follows closely the line of Marcal et al. ${ }^{5,6)}$; Method II, the modified $r_{\min }$ method of Inoue et al. ${ }^{12)}$; Method III, the author's modification of the $r_{\min }$ method. The author also presented an algorithm for determining time increment, demonstrating its simplicity and effectiveness. As a test problem, the analysis was performed on centrifugal casting mold, and the computational results obtained by the above three methods were compared with each other in terms of the "followability" of the equivalent stress and the flow stress of the material.

Chapter III of the paper deals with the problems of bore surface cracking and mold warping or bowing of centrifugal casting mold. Although there have been some attempts so far to obtain mechanic solutions of this kind,14,15) they are, as far as centrifugal casting is concerned, mostly thermoelastic solutions. ${ }^{14)}$

Therefore, the author studied the influences of casting parameters on the thermo-elastic-plastic behaviors of mold during centrifugal casting.

The mold and ingot materials dealt with in this paper are $0.4 \%$ carbon steel and $\mathrm{HK} 40(0.4 \mathrm{C}-25 \mathrm{Cr}-$ $20 \mathrm{Ni}$ heat resistant alloy), respectively.

\section{A Study of the Solution Methods for Thermo- elastic-Plastic FEM}

\section{Theoretical Formulations}

The stress-strain relations or the constitutive relations during plastic deformation were first given by the Levy-Mises-Prandtl-Reuss equations, which are concisely dealt with by Hill. ${ }^{16)}$ Later, McGlintock ${ }^{17)}$ provided a physical insight into these relations introducing the dislocation mechanisms involved in plastic deformation.

The derivations in this paper are based on the plasticity theories of Yamada, ${ }^{3,4)}$ into which the tem- 
perature dependencies of thermal expansion coefficient and Young's modulus are incorporated. Isotropic hardening is assumed. The incremental form of the resulting constitutive equations in thermo-elasticplastic deformation can be expressed as

$$
\begin{array}{r}
\{d \sigma\}=\left[D^{\mathrm{p}}\right]\left(\{d \varepsilon\}-\{\alpha\} d T-\left\{\frac{\partial \alpha}{\partial T}\right\} T d T-\frac{\partial\left[D^{\mathrm{e}}\right]^{-1}}{\partial T} .\right. \\
\{\sigma\} d T)-\frac{1}{S}\left[D^{\mathrm{e}}\right]\left\{\frac{\partial f}{\partial \sigma}\right\} \frac{\partial f}{\partial T} d T \ldots \ldots \ldots . .(1)
\end{array}
$$

where, $f$ : yield function

$\alpha$ : thermal expansion coefficient

$\left[D^{\mathrm{e}}\right],\left[D^{\mathrm{p}}\right]$ : the elasticity and plasticity matrices, respectively, the scalar $S=3 G$ $+H^{\prime}$ with the adoption of the Mises yield criterion

$G$ : the shear modulus of elasticity

$H^{\prime}=\partial H / \partial \bar{\varepsilon}^{\mathrm{p}}$ : the slope of flow stress $\bar{H}$-plastic strain $\bar{\varepsilon}^{\mathrm{p}}$ diagram in uniaxial tensile test.

The term $\partial\left[D^{\ominus}\right]^{-1} / \partial T \cdot\{\sigma\} d T$ represents the temperature dependency of the modulus of elasticity, and the term $\left[D^{\circ}\right]\{\partial f / \partial \sigma\} \cdot \partial f / \partial T \cdot d T / S$ is the temperature dependency of the yield function. Adopting the Mises yield criterion as mentioned, the yield function is given by Eq. (2) in which the flow stress for an isotropic material is assumed to be uniquely determined by the accumulated equivalent plastic strain so far and the current temperature.

$$
\left.\begin{array}{rl}
f= & \frac{1}{\sqrt{2}}\left[\left(\sigma_{x}-\sigma_{y}\right)^{2}+\left(\sigma_{y}-\sigma_{z}\right)^{2}+\left(\sigma_{z}-\sigma_{x}\right)^{2}\right. \\
& \left.+6\left(\tau_{x y}^{2}+\tau_{y z}^{2}+\tau_{z x}^{2}\right)\right]^{1 / 2}-\bar{H}\left(\int d \bar{\varepsilon}^{\mathrm{p}}, T\right) \\
\text { or } \quad f= & \bar{\sigma}-\bar{H}\left(\int d \bar{\varepsilon}^{\mathrm{p}}, T\right)
\end{array}\right\}
$$

$\bar{H}$ is assummed to be given by $\bar{H}=\bar{H}_{0}+H^{\prime} \bar{\varepsilon}^{\mathrm{p}}$ where $\bar{H}_{0}$ is the initial yield stress.

The components of plastic strain increments are obtained from the associated flow rule as

$$
\left\{d \varepsilon^{\mathrm{p}}\right\}=d \lambda\left\{\frac{\partial f}{\partial \sigma}\right\}
$$

where the scalar proportionality is given by

$$
\begin{aligned}
d \lambda= & d \bar{\varepsilon}^{\mathrm{p}}=\left\{\frac { 3 G } { \overline { \sigma } } \left(S_{x} d \varepsilon_{x}+S_{y} d \varepsilon_{y}+S_{z} d \varepsilon_{z}+\tau_{x y} d \gamma_{x y}\right.\right. \\
& \left.\left.+\tau_{y z} d \gamma_{y z}+\tau_{z x} d \gamma_{z x}\right)+\frac{\bar{\sigma}}{E} \frac{\partial E}{\partial T} d T-\frac{\partial \bar{H}}{\partial T} d T\right\} / \\
& \left(3 G+H^{\prime}\right) \quad \ldots \ldots \ldots \ldots \ldots \ldots \ldots \ldots \ldots \ldots \ldots \ldots \ldots \ldots \ldots \ldots \ldots \ldots \ldots \ldots \ldots \ldots \ldots \ldots \ldots \ldots
\end{aligned}
$$

$E$ is Young's modulus and $S_{x}, \ldots$ are deviatoric stresses defined by $S_{i j}=\sigma_{i j}-\frac{1}{3} \delta_{i j} \sigma_{m m}$ with $\delta_{i j}$ being Kronecker's delta. $d \gamma_{x y}, \ldots$ are engineering shear strain increments.

Applying the principles of virtual work to Eq. (1), equilibrium equations of an element and then over a structure after usual assembling of FEM are expressed as

$$
\begin{aligned}
\{\Delta L\}_{\text {external }}= & {[K]\{\Delta U\}-\{\Delta L\}_{t h}-\{\Delta L\}_{q} } \\
& -\{\Delta L\}_{E}-\{\Delta L\}_{f} \ldots \ldots \ldots \ldots \ldots \ldots
\end{aligned}
$$

where, $[K]$ : stiffness matrix over a structure $\{\Delta U\}:$ nodal displacement increments $\{\Delta L\}$ : nodal load increments.

the suffix ' external' designates the external force, and the subscripts th, $q, E$ and $f$ denote the influences due to thermal expansion and to the temperature dependencies of $\alpha, E$ and yield function $f$, respectively. The above equation was obtained by Inoue et al. ${ }^{12,18)}$ and the term $\{\Delta L\}_{E}$ by Ueda and Yamakawa. ${ }^{19)}$

\section{Algorithm to Determine Time Increment}

In problems dealing with thermal stresses, the choice of the time increments (hence, the magnitude of thermal load increments) is the key to the solution accuracy and stability because the degree of stress increments as well as the values of various temperature dependent material properties are determined by the time step size.

In this connection, there is a possibility that, when the $r_{\text {min }}$ method is employed, excessively large time increment might be required to yield a next element, thus leading to the increase of errors. In such a case, to avoid this the time step size should somehow be sub-incremented or decreased.

In addition, in heat treatment processes such as quenching and casting, the temperature variations are considerably rapid at the beginning and become quite sluggish as time proceeds. Therefore, if there is no algorithm for determining the time increments, trials and errors of time increment strategy are inevitable and a considerable amount of time and labor would be required before obtaining a reasonable solution and the overall running cost can be very expensive. To solve these problems, a simple but efficient algorithm has been introduced, which is described below.

Now, let us consider a certain load step $i$ with $n$ elements. Let $\Delta T_{1}, \Delta T_{2}, \ldots, \Delta T_{n}$ be the distribution of temperature increments from the previous load step $i-1$ at time $t_{j}$ where $t_{j}$ corresponds to $j$-th time step in the $i$-th load step and $\left(\Delta T_{\max }\right)_{j}$ be the maximum temperature increment. The times $t_{1}, t_{2}, \ldots t_{i}$, . . . in the $i$-th load step are automatically determined from the stability criteria for the finite difference forms of heat flow equation. ${ }^{21)}$ Also, let us assume that the max. temperature increment in the next time step $t_{j+1}$ exceeds a given temperature increment DTMAX; thus,

$$
\left|\Delta T_{\max }\right|_{j}<\mathrm{DTMAX} \leqq\left|\Delta T_{\max }\right|_{j+1} .
$$

The time for the $i$-th load step is determined by assuming local linearization so that,

$$
\begin{aligned}
& t_{i}=t_{j}+m\left(t_{j+1}-t_{j}\right), \quad 0 \leqq m \leqq 1 \\
& \text { where, } \quad m=\frac{\text { DTMAX }-\left|\Delta T_{\max }\right|_{j}}{\left|\Delta T_{\max }\right|_{j+1}-\left|\Delta T_{\max }\right|_{j}}
\end{aligned}
$$

The temperature distribution at $t_{i}$ is then obtained by scaling with $m$. If the time step sizes within the 
load step are small enough compared with $\Delta t_{i}\left(\equiv t_{i}-\right.$ $\left.t_{i-1}\right), t_{i}$ may simply be chosen such that $t_{i}=t_{j+1}$.

This simpler algorithm is used throughout this paper. As will be seen afterwards, the above algorithm considerably reduces the number of thermal load increments. This may be compared with Ref. 20 ), where residual stress analysis on quenching was done with as many thermal loading steps as 430 .

\section{Test Problem}

Thermal stress analysis of centrifugal casting mold was performed as a test problem. The calculation of temperature was performed by finite difference method. The basic equations and material properties used for the analysis are available in Ref. 21). However, the method described therein has been modified to read in arbitrarily meshed coordinates in radial direction in accordance with finite element mesh configuration.

The casting conditions are given in Table 1 and the finite element mesh configuration is shown in Fig. 1. Data from Inoue and Tanaka ${ }^{18)}$ were used with the flow stress of $0.4 \% \mathrm{C}$ steel mold, and the other material properties are available in Handbook of Steel Materials (ed. by ISIJ in 1967). Plotted in Fig. 2 are the temperature histories of elements $v s$. the number of thermal load increments. As can be seen, "smooth" temperature increments were obtained (DTMAX $=20^{\circ} \mathrm{G}$ ). The timings of the completion of solidification and the removal of tube and the duration of water-cooling on the mold O.D. (outer diameter) were also marked. After $700 \mathrm{sec}$, the temperature distribution became almost uniform, approximately $290^{\circ} \mathrm{C}$. The number of thermal

Table 1. Centrifugal casting conditions.

\begin{tabular}{l|l} 
Casting temperature & $1420{ }^{\circ} \mathrm{C}$ \\
Preheating temperature of mold & $280{ }^{\circ} \mathrm{C}$ \\
Coating thickness & $0.15 \mathrm{~cm}$ \\
Thermal conductivity of coating & $0.01 \mathrm{cal} / \mathrm{cm} \cdot \mathrm{sec} \cdot{ }^{\circ} \mathrm{C}$ \\
Wall-thickness of tube & $2.0 \mathrm{~cm}$ \\
Timing of the removal of tube & $180 \mathrm{sec}$ \\
Water-spraying conditions* & \\
Duration & $240 \sim 480 \mathrm{sec}$ \\
Heat transfer coefficient $\left(h_{w}\right)$ & $0.01 \mathrm{cal} / \mathrm{cm}^{2} \cdot \mathrm{sec} \cdot{ }^{\circ} \mathrm{C}$
\end{tabular}

* Heat flow rate from mold surface is calculated by $q=h_{w}\left(T_{s}\right.$ $-T_{w}$ ), where $T_{s}$ and $T_{w}$ are the surface and water temperatures, respectively.
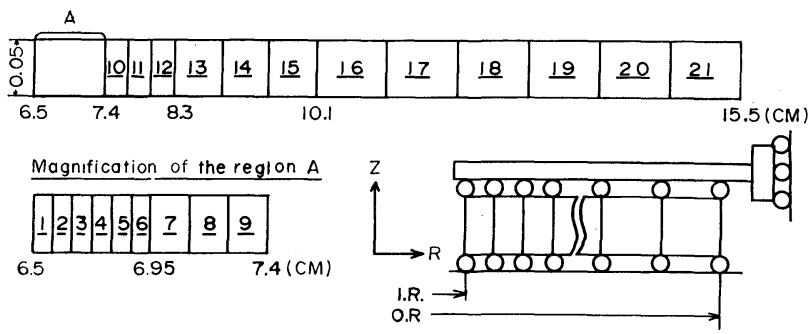

Fig. 1. Finite element mesh configuration and generalized plane strain boundary condition. load increments required was 38. Accordingly, the thermal stress analysis was done for one cycle operation of $700 \mathrm{sec}$ under generalized plane strain condition, i.e., $\varepsilon_{z}=$ const.

\section{Method I: Effective Stiffness Method}

Now, let us consider a thermal load increment $D T=T_{2}-T_{1}$ and assume the local linearization of temperature, stresses, strains, etc. After calculating the scalar $r$ defined by Eq. (8) which is the fraction of the equivalent stress increment up to the yield point over the "trial" equivalent stress increment $D \bar{\sigma}$, the stresses, strains and temperature at the beginning of plastic deformation are determined as follows:

$$
\begin{aligned}
& \mathcal{T}_{r}=\mathcal{T}_{1}+r \cdot D T \quad(0 \leqq r \leqq 1) \\
& \{\varepsilon\}_{r}=\{\varepsilon\}_{1}+\{\Delta \varepsilon\}_{r}, \quad\{\varepsilon\}_{r}=r\{\Delta \varepsilon\} \\
& \{\sigma\}_{r}=\{\sigma\}_{1}+\{\Delta \sigma\}_{r}, \quad\{\Delta \sigma\}_{r}=r\{D \sigma\}
\end{aligned}
$$

The value of $r$ may be obtained from

$$
f_{T^{\prime}=T^{r}}=f_{T^{\prime}=T_{1}}+r d f=0
$$

along with Eq. (2), as

$$
r=-\begin{gathered}
(\bar{\sigma}-\bar{H})_{T^{\prime}=T_{r}} \\
D \sigma-\frac{\partial \bar{H}}{\partial T} D T
\end{gathered}
$$

Dividing the straining during $D T$ into elastic and elastoplastic parts in the straightforward manner after Snyder and Bathe, ${ }^{10)}$ the constitutive equations or the stress increments can be expressed as the sum of $\{\Delta \sigma\}_{r}$ for the elastic deformation during $\mathcal{T}_{r}-\mathcal{T}_{1}$ and $\{\Delta \sigma\}_{2}$ for the elastoplastic deformation during the remaining part of $T_{2}-T_{r}$. The result is

$$
\left.\begin{array}{rl}
\{\Delta \sigma\}= & {\left[D^{\mathrm{err}}\right]\left(\{\Delta \varepsilon\}-\{\alpha\} D T-\left\{\frac{\partial \alpha}{\partial T}\right\} \mathcal{T}_{1} D T\right.} \\
& \left.-\frac{\partial\left[D^{\mathrm{e}}\right]^{-1}}{\partial T}\{\sigma\}_{1} D T\right)+\frac{3 G}{\left(3 G+H^{\prime}\right) \bar{\sigma}_{r}} \frac{\partial \bar{H}}{\partial T} \\
& \times\left\{\sigma^{\prime}\right\}\left(\mathcal{T}_{2}-T_{r}\right)
\end{array}\right\}
$$

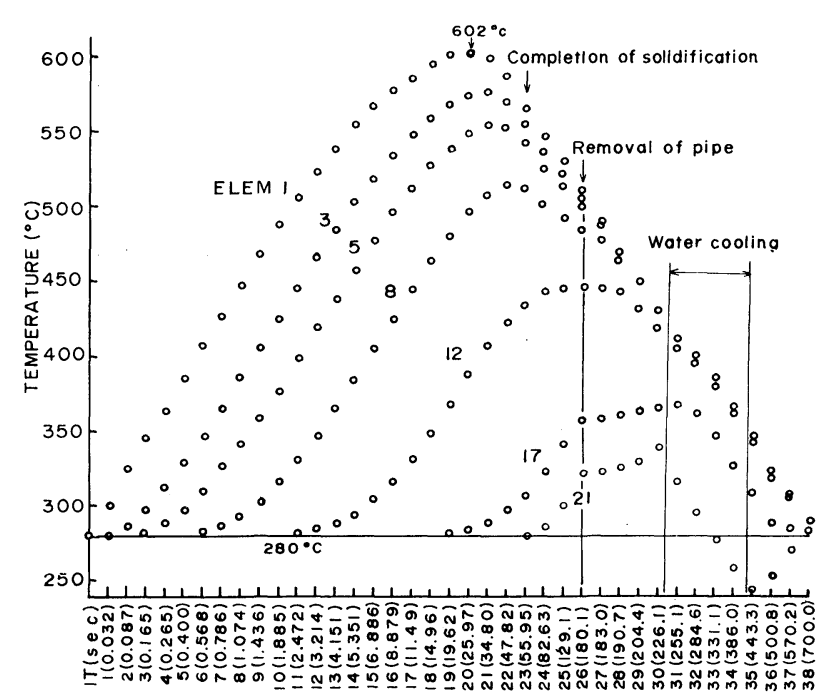

Fig. 2. Temperature histories of the elements plotted against the iteration number of thermal loading. 
where,

$$
\begin{gathered}
{\left[D^{\mathrm{eff}}\right]=r\left[D^{\ominus}\right]+(1-r)\left[D^{\mathrm{p}}\left(\sigma_{r}\right)\right]} \\
\sigma^{\prime}: \quad \text { deviatoric stresses }
\end{gathered}
$$

subscript $r$ : the values at $T=T_{r}$

$\left[D^{\text {eff }}\right]$ is same as "mean stiffness coefficient" given by Marcal et al. ${ }^{5,6)}$ but here is called " effective stiffness matrix".

The equilibrium equation over a structure is

$$
\begin{aligned}
\{\Delta L\}_{\text {external }}= & {\left[K^{\text {err }}\right]\{\Delta U\}-\{\Delta L\}_{t h}-\left\{\Delta L_{q}\right\} } \\
& -\{\Delta L\}_{E}-\{\Delta L\}_{f} \ldots \ldots \ldots \ldots \ldots \ldots \ldots \ldots \ldots
\end{aligned}
$$

where the "effective stiffness matrix over a structure", $\left[K^{\text {eff }}\right]$ consists of $\left[D^{\text {aff }}\right]$ instead of $\left[D^{\mathrm{p}}\right]$. Initially estimated strain increments $\left\{\Delta_{\varepsilon}\right\}$ are not obviously correct, but it is expected that the use of the resulting stiffness matrix $\left[D^{\text {eff }}\right]$ for the solution of Eq. (10) gives a better estimates of $\left\{\Delta_{\varepsilon}\right\}$. Modified Newton-Raphson algorithm ${ }^{1,2)}$ uses the initially estimated $\left[D^{\text {eff }}\right]$ and hence $\left[K^{\text {efr }}\right]$ until the convergency is obtained. The convergency was checked within $0.5 \%$ error over the thermal load increments. The rate of convergency was significantly fast; typically 3 iterations for plasticity-continuing elements and 3 to 8 for elastic-plastic transitional elements.

The results thus obtained are given in Table 2 in which the "errors" between the calculated equivalent stress $\bar{\sigma}$ and the flow stress $\bar{H}$ are presented. These errors or the followability of $\bar{\sigma}$ and $\bar{H}$ are considered to be an important measure for evaluating the reliability of the solution method. All input data are given in Sec. II. 3, excepting that DTMAX was taken $10^{\circ} \mathrm{C}$. Complete solutions are seen in Figs. 4 and 5. There were negligible errors with Elements 1 and 2. In Element 2, however, its stress distributions of other components (not shown here) were not "smooth", obviously disturbing the solution. After Element 3, the errors became fairly large and the

\begin{tabular}{|c|c|c|c|c|c|c|}
\hline \multirow{2}{*}{$\begin{array}{c}I T P \\
\left(\begin{array}{c}\text { Time } \\
(\mathrm{sec})\end{array}\right)\end{array}$} & \multirow{2}{*}{$\begin{array}{l}\bar{\sigma}, \bar{H}, \\
\text { Error }\end{array}$} & \multirow{2}{*}{$\begin{array}{c}\text { Number } \\
\text { of itera- } \\
\text { tions }\end{array}$} & \multicolumn{4}{|c|}{ Element No. } \\
\hline & & & 1 & 2 & 3 & 4 \\
\hline $\begin{array}{c}1 \\
(0.098)\end{array}$ & $\begin{array}{l}\bar{\sigma}\left(\mathrm{kg} / \mathrm{cm}^{2}\right) \\
\bar{H}\left(\mathrm{~kg} / \mathrm{cm}^{2}\right) \\
\text { Error }(\%)\end{array}$ & 5 & $\begin{array}{c}1882 \\
1882 \\
-\end{array}$ & & & \\
\hline $\begin{array}{c}5 \\
(0.254)\end{array}$ & ” & 3 & $\begin{array}{c}1896 \\
1896 \\
-\end{array}$ & $\begin{array}{c}1874 \\
1874 \\
-\end{array}$ & & \\
\hline $\begin{array}{c}7 \\
(0.400)\end{array}$ & $"$ & 8 & $\begin{array}{c}1902 \\
1902 \\
-\end{array}$ & $\begin{array}{c}1882 \\
1882 \\
-\end{array}$ & $\begin{array}{r}1993 \\
1873 \\
(6.41)\end{array}$ & \\
\hline $\begin{array}{c}9 \\
(0.625)\end{array}$ & ", & 7 & $\begin{array}{c}1904 \\
1904 \\
-\end{array}$ & $\begin{array}{c}1890 \\
1890 \\
-\end{array}$ & $\begin{array}{r}2002 \\
1882 \\
(6.38)\end{array}$ & $\begin{array}{r}2107 \\
1874 \\
(12.4)\end{array}$ \\
\hline
\end{tabular}

Table 2. Errors between calculated equivalent stress $\bar{\sigma}$ anc flow stress $\bar{H}$ of the elements in thermoelastoplastic state (Method I).

Note: ITP $=$ number of load increments after first yielding. Error $=\frac{\bar{\sigma}-\bar{H}}{\bar{H}} \times 100(\%)$ iteration was stopped at 10. In another example for which a cyclic temperature history was given (after 26 sec of Fig. 2), yielding judgement failed in unloading and subsequent reloading processes because of these growing errors, and the solution became unstable.

Thus, despite that the thermal load increments were small enough to allow the elements to yield one by one and that the solution is considered to have converged the iterative convergent method using the effective stiffness matrix defined by Eq. (8) failed to obtain a reliable solution.

\section{Method II: Modified $r_{\min }$ Method I}

As already mentioned, Inoue et al. ${ }^{12)}$ modified the $r_{\mathrm{min}}$ method of Yamada et al. ${ }^{11)}$ to apply to thermal stress analysis, which states as follows:

(1) For a thermal load increment $D T=T_{2}-T_{1}, n$ elements are assumed to yield. For these elements, a series of the scalars $r$ (as defined in this paper by Eq. (8)) are calculated and are arranged in the order of magnitude so that

$$
r_{1}<r_{2}<r_{3} \cdots \cdots<r_{n}
$$

(2) The next step is to increase the temperature distributions step by step by $D T r_{2}, D T\left(r_{2}-r_{1}\right)$, $D T\left(r_{3}-r_{2}\right), \ldots$, and $D T\left(1-r_{n}\right)$, and recalculations are made; in doing so, the elements yield one by one. If the $n+1$-th calculation is over, we return to step (1) for the next load increment.

The results obtained by the above algorithm are shown in Table 3 in terms of the errors between $\bar{\sigma}$ and $\bar{H}$. DTMAX was taken $30^{\circ} \mathrm{C}$ large enough to assure a simultaneous yielding of more than one element. The magnitude of DTMAX does not affect the temperature distribution. Now, we shall study what happened in the load increment step 4 to 5 in detail where Elements 4 and 5 simultaneously yielded. In Fig. 3(a) the loading steps and material responses are schematically illustrated ignoring for brevity the temperature dependencies of Young's modulus and initial yield stress $\bar{H}_{0}$. For the first step $D T, r_{1}$ was 0.107 and $r_{2}$ was 0.885 and hence $H_{0}$ were 1873 and $1870\left(\mathrm{~kg} / \mathrm{cm}^{2}\right)$, respectively, for Elements 4 and 5 . For $D T r_{1}$, recalculation was not actually done, but solution was scaled with $r_{1}$ so that $\bar{\sigma}=\bar{\sigma}$ (previous) $+D \bar{\sigma} \cdot r_{1}=1762+1034 \times 0.107=1873$ for Element 4 and similarly $\bar{\sigma}=1.167$ for Element 5 . As a matter of course, $\bar{\sigma}$ coincides with $\bar{H}_{0}$ for Element 4 . For the second step of $D T\left(r_{2}-r_{1}\right)$, recalculation was done regarding Element 4 as an elastic-plastic element, and $\bar{\sigma}$ was calculated to be 1886 for Element 5 which does not coincide with the initial yield stress $\bar{H}_{0}$ $(=1870)$. This discrepancy in turn gives rise to the error of $0.75 \%$ in the final step of $D T\left(1-r_{2}\right)$.

Thus, when more than one element yield simultaneously, difficulty occurs in estimating initial yield stresses in those elements other than $r_{\text {min }}$ element, and errors are inevitable. This is because the stress states in those elements are affected by "softening" due to yielding of the neighboring elements. In other 
Table 3. Errors between equivalent stress $\bar{\sigma}$ and flow stress $\bar{H}$ of the elements in thermoelastoplastic state (Method II).

\begin{tabular}{|c|c|c|c|c|c|c|c|c|c|c|c|c|c|c|}
\hline \multirow{2}{*}{$\begin{array}{c}I T \\
\left(\begin{array}{c}\text { Time } \\
(\mathrm{sec})\end{array}\right)\end{array}$} & \multicolumn{14}{|c|}{ Element No., $\bar{\sigma}\left(\mathrm{kg} / \mathrm{cm}^{2}\right)$ and Errors (\%) } \\
\hline & 1 & 2 & 3 & 4 & 5 & 6 & 7 & 8 & 9 & 10 & 11 & 12 & 13 & $14 \cdots \cdots$ \\
\hline $\begin{array}{c}2 \\
(0.154)\end{array}$ & $\begin{array}{c}1888 \\
-\end{array}$ & & & & & & & & & & & & & \\
\hline $\begin{array}{c}3 \\
(0.310)\end{array}$ & $\begin{array}{c}1899 \\
-\end{array}$ & $\begin{array}{c}1885 \\
-\end{array}$ & & & & & & & & & & & & \\
\hline $\begin{array}{c}4 \\
(0.546)\end{array}$ & $\begin{array}{r}1903 \\
-0.10\end{array}$ & $\begin{array}{c}1895 \\
-\end{array}$ & $\begin{array}{c}1883 \\
-\end{array}$ & & & & & & & & & & & \\
\hline $\begin{array}{c}5 \\
(0.889)\end{array}$ & $\begin{array}{r}1898 \\
-0.11\end{array}$ & $\begin{array}{c}1901 \\
-\end{array}$ & 1893 & $\begin{array}{c}1882 \\
-\end{array}$ & $\begin{array}{l}1885 \\
(0.75)\end{array}$ & & & & & & & & & \\
\hline $\begin{array}{c}6 \\
(1.377)\end{array}$ & $\begin{array}{r}1877 \\
-0.21\end{array}$ & $\begin{array}{r}1897 \\
-0.10\end{array}$ & $\begin{array}{c}1898 \\
-\end{array}$ & $\begin{array}{c}1891 \\
-\end{array}$ & $\begin{array}{l}1895 \\
(0.80)\end{array}$ & $\begin{array}{c}1872 \\
-\end{array}$ & & & & & & & & \\
\hline $\begin{array}{c}7 \\
(2.076)\end{array}$ & $\begin{array}{r}1867 \\
-0.32\end{array}$ & $\begin{array}{r}1880 \\
-0.16\end{array}$ & $\begin{array}{r}1895 \\
-0.11\end{array}$ & $\begin{array}{c}1896 \\
-\end{array}$ & $\begin{array}{l}1904 \\
(0.79)\end{array}$ & $\begin{array}{c}1882 \\
-\end{array}$ & $\begin{array}{l}1870 \\
-\end{array}$ & & & & & & & \\
\hline $\begin{array}{c}8 \\
(3.091)\end{array}$ & $\begin{array}{r}1824 \\
-0.55\end{array}$ & $\begin{array}{r}1868 \\
-0.32\end{array}$ & $\begin{array}{r}1877 \\
-0.21\end{array}$ & $\begin{array}{r}1891 \\
-0.11\end{array}$ & $\begin{array}{l}1906 \\
(0.69)\end{array}$ & $\begin{array}{c}1889 \\
-\end{array}$ & $\begin{array}{r}1880 \\
0.11\end{array}$ & $\begin{array}{r}1865 \\
0.11\end{array}$ & & & & & & \\
\hline $\begin{array}{c}9 \\
(4.536)\end{array}$ & $\begin{array}{c}1766 \\
(-0.73)\end{array}$ & $\begin{array}{r}1832 \\
-0.49\end{array}$ & $\begin{array}{r}1867 \\
-0.32\end{array}$ & $\begin{array}{r}1871 \\
-0.16\end{array}$ & $\begin{array}{r}1898 \\
0.64\end{array}$ & $\begin{array}{c}1889 \\
-\end{array}$ & 1885 & $\begin{array}{r}1874 \\
0.16\end{array}$ & $\begin{array}{r}1860 \\
0.16\end{array}$ & & & & & \\
\hline $\begin{array}{l}10 \\
(6.631)\end{array}$ & $\begin{array}{c}1707 \\
(-0.87)\end{array}$ & $\begin{array}{r}1777 \\
-0.67\end{array}$ & $\begin{array}{r}1831 \\
-0.49\end{array}$ & $\begin{array}{r}1864 \\
-0.32\end{array}$ & $\begin{array}{r}1874 \\
0.29\end{array}$ & $\begin{array}{c}1877 \\
-\end{array}$ & $\begin{array}{c}1884 \\
-\end{array}$ & $\begin{array}{r}1879 \\
0.11\end{array}$ & $\begin{array}{r}1869 \\
0.21\end{array}$ & $\begin{array}{r}1852 \\
0.22\end{array}$ & & & & \\
\hline $\begin{array}{l}12 \\
(9.723)\end{array}$ & $\begin{array}{c}1657 \\
(-0.90)\end{array}$ & $\begin{array}{r}1725 \\
-0.69\end{array}$ & $\begin{array}{r}1781 \\
-0.56\end{array}$ & $\begin{array}{r}1826 \\
-0.38\end{array}$ & $\begin{array}{r}1872 \\
0.54\end{array}$ & $\begin{array}{r}1865 \\
-0.16\end{array}$ & $\begin{array}{c}1870 \\
-\end{array}$ & $\begin{array}{r}1878 \\
0.16\end{array}$ & $\begin{array}{r}1874 \\
0.27\end{array}$ & $\begin{array}{r}1863 \\
0.54\end{array}$ & $\begin{array}{r}1844 \\
0.55\end{array}$ & & & \\
\hline $\begin{array}{c}13 \\
(21.653)\end{array}$ & $\begin{array}{r}1608 \\
-0.62\end{array}$ & $\begin{array}{r}1657 \\
-0.54\end{array}$ & $\begin{array}{r}1702 \\
-0.47\end{array}$ & $\begin{array}{r}1743 \\
-0.29\end{array}$ & $\begin{array}{r}1793 \\
0.67\end{array}$ & $\begin{array}{r}1807 \\
0.11\end{array}$ & $\begin{array}{c}1840 \\
-\end{array}$ & $\begin{array}{r}1855 \\
0.11\end{array}$ & $\begin{array}{r}1853 \\
0.32\end{array}$ & $\begin{array}{r}1861 \\
0.59\end{array}$ & $\begin{array}{l}1867 \\
(1.74)\end{array}$ & $\begin{array}{r}1847 \\
1.60\end{array}$ & & \\
\hline $\begin{array}{c}14 \\
(33.232)\end{array}$ & $\begin{array}{r}1619 \\
-0.74\end{array}$ & $\begin{array}{r}1660 \\
-0.36\end{array}$ & $\begin{array}{r}1694 \\
-0.30\end{array}$ & $\begin{array}{r}1725 \\
-0.23\end{array}$ & $\begin{array}{r}1768 \\
0.74\end{array}$ & $\begin{array}{r}1777 \\
-0.17\end{array}$ & $\begin{array}{c}1807 \\
-\end{array}$ & $\begin{array}{c}1836 \\
-\end{array}$ & $\begin{array}{l}1843 \\
0.22\end{array}$ & $\begin{array}{l}1840 \\
0.55\end{array}$ & $\begin{array}{c}1857 \\
(1.75)\end{array}$ & $\begin{array}{l}1841 \\
1.60\end{array}$ & $\begin{array}{l}1809 \\
0.95\end{array}$ & \\
\hline $\begin{array}{c}15 \\
(54.327)\end{array}$ & $\longleftarrow$ & & & nloading & & & $\longrightarrow$ & $\begin{array}{r}1826 \\
0.39\end{array}$ & $\begin{array}{r}1834 \\
0.38\end{array}$ & $\begin{array}{r}1826 \\
0.66\end{array}$ & $\begin{array}{l}1835 \\
(1.77)\end{array}$ & $\begin{array}{r}1825 \\
1.73\end{array}$ & $\begin{array}{r}1807 \\
1.75\end{array}$ & \\
\hline $\begin{array}{c}16 \\
(96.010)\end{array}$ & & & & & & & & $\longleftarrow$ & & unloadir & & $\longrightarrow$ & $\begin{array}{r}1789 \\
2.05\end{array}$ & $\begin{array}{l}1771 \\
(2.25)\end{array}$ \\
\hline $\begin{array}{c}17 \\
(174.76)\end{array}$ & $\begin{array}{r}2237 \\
0.44\end{array}$ & $\begin{array}{c}2485 \\
(14.36)\end{array}$ & & & & & & & & & & unloading & $\longleftrightarrow$ & $\begin{array}{r}1743 \\
2.83\end{array}$ \\
\hline $\begin{array}{c}18 \\
(184.710)\end{array}$ & $\begin{array}{c}2312 \\
(-8.80)\end{array}$ & $\begin{array}{r}2553 \\
6.02\end{array}$ & $\begin{array}{r}2197 \\
-5.95\end{array}$ & $\begin{array}{r}2147 \\
-4.62\end{array}$ & & & & & & & & & & $\begin{array}{r}1744 \\
2.83\end{array}$ \\
\hline
\end{tabular}

Note: $I T=$ number of load increments.

( ) =maximum error. No yielding element at $I T=11$.

words, the material response differs between the first "trial" load step and the subsequent steps, and therefore the scalars $r_{2}, r_{3} \ldots$ are no longer correct excepting $r_{1}$ or $r_{\min }$. This trend is more prominent with Element 2 in the step of 16 to 17 where the error is as large as $14 \%$. After 18 steps, the solution was disturbed and became unstable.

It is, therefore, concluded that in the strict sense this algorithm does not allow elements to yield one by one and that if one performs it literally, errors which may be acceptable in a practical sense are unavoidable. This is particularly so when thermal loading history is complicated as in the case of this example. Besides, the number of recalculations required for a load increment is same as that in the other modified method, as seen in Fig. 3, which will
Error $=\frac{\bar{\sigma}-\bar{H}}{\bar{H}} \times 100(\%)$.

Upper figures denote $\bar{\sigma}$ and lower figures error.

be described in the following sub-section.

\section{Method III: Modified $r_{\min }$ Method II}

In this version of the modified $r_{\min }$ method, thermal load increments are adjusted strictly so as to yield elements one by one within prescribed thermal loadings. The algorithm is simple and is stated as follows:

(1) With $n$ yielding elements for a thermal load increment $D T=T_{2}-T_{1}$, the scalars $r_{1}, r_{2}, \ldots r_{n}$ are calculated, $r_{\min }$ being the minimum scalar.

(2) The solution at $T=T_{r_{\min }}$ is obtained by scaling with $r_{\mathrm{min}}$, and the thermal loading step is proceeded up to $T=T_{r_{m i n}}$. Then, we return to (1) taking the load increment as $T_{2}-T_{r_{\min }}$ and regarding the $r_{\min }$ element as thermoelastoplastic, thus remaining $(n-1)$ elements to be yielded in the next step. 


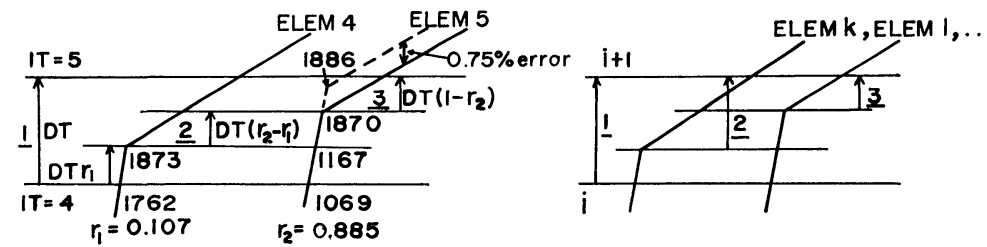

(a) Modified $r_{\text {min }}$ method (I)

(b) Modified $r_{\text {min }}$ method (II)

Fig. 3. Schematic illustrations of two modified $r_{\min }$ methods.

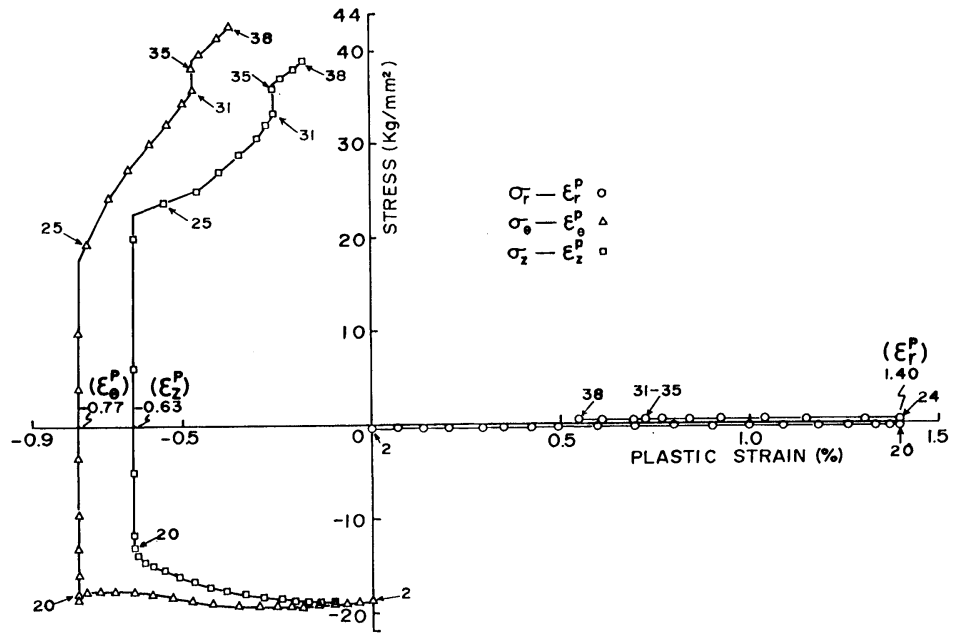

Arrows indicate iteration numbers.

Fig. 5. Stress-plastic strain cycles of Element 1.

The above is repeated until all elements yield. A schematic illustration of the algorithm is also included in Fig. 3(b) where the temperature dependencies are omitted for simplicity.

\section{Thermal Stress State}

The results obtained with DTMAX $=20^{\circ} \mathrm{G}$ will be shown below before discussing errors. Figure 4(a) to (c) show the distributions of stress components at various time steps. When the I.D. (inner diam., Element 1) reaches the max. temperature of $602{ }^{\circ} \mathrm{C}$ after $26 \mathrm{sec}$, the plastic zone reaches $2.1 \mathrm{~cm}$ from the inside; thus about one fourth of the wall thickness undergoes plastic deformation. As temperature decreases in the inner region, unloading occurs in this region and the stress state changes from compression to tension. As time further proceeds, this region again undergoes plastic deformation.

The above deformation process is better seen by the stress-plastic strain cycle of Element 1 having the max. stress amplitude, Fig. 5. That is, the mode of the plastic deformation in the inner element is compressive in both the circumferential and axial directions and protrusive in the radial direction and vice versa during the cooling period.

It is interesting to note that the sudden unloading and subsequent thermoelastic deformation took place during water cooling period (Steps 31 to 35 ).

It is also worthwhile to mention that the plastic tip zone does not remain about one fourth of the wall thickness, as seen in Fig. 4(a), but it proceeds further outward while unloading occurs in the inner elements and that as a result of this, the number of elasticplastic boundaries is not necessarily one but can be

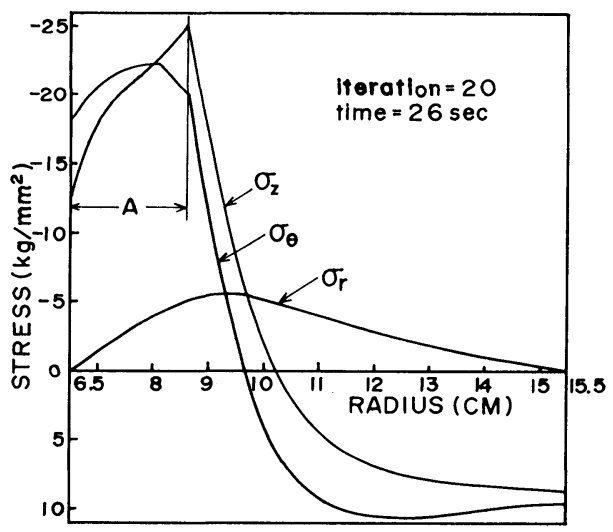

(a): $I T=20(26 \mathrm{sec})$

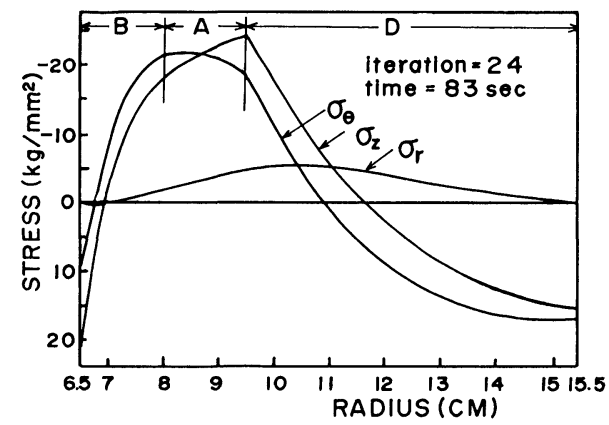

(b): $I T=24(83 \mathrm{sec})$

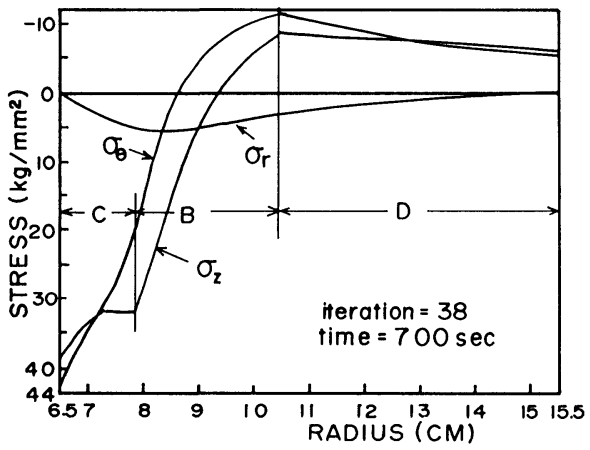

(c): $I T=38(700 \mathrm{sec})$

$\Lambda=$ yiclded and plasticity-continuing regions $\mathrm{B}=$ unloaded elastic region

$\mathrm{C}=$ plastic region re-yielded from the state $\mathrm{B}$ $\mathrm{D}=$ consistently elastic region

Fig. 4. Stress distributions at various load steps.

two or more.

\section{Errors between Equivalent Stress and Flow Stress}

As is seen in Table 4, the overall errors with this method are exceedingly small compared with the other two methods discussed earlier. Errors less than $1 \%$ have been accomplished up to $25 \mathrm{sec}$ (20 iterations) at which the thermal load became maximum and after $130 \mathrm{sec}$ (25 iterations) errors are typically 1 to $2 \%$. In the steps of 35 to 36 , ten elements yielded simultaneously and negligibly small errors emerged (i.e., of the order of $0.0 n \%$ ). The maximum error throughout the complete cycle of thermal loading was only $2.28 \%$. As can be seen, the errors grow slightly larger in roughly meshed elements. These errors can be reduced by suitably "balancing" 
Table 4. Errors between equivalent stress $\bar{\sigma}$ and flow stress $\bar{H}$ of the elements in thermoelastoplastic state (Method III)

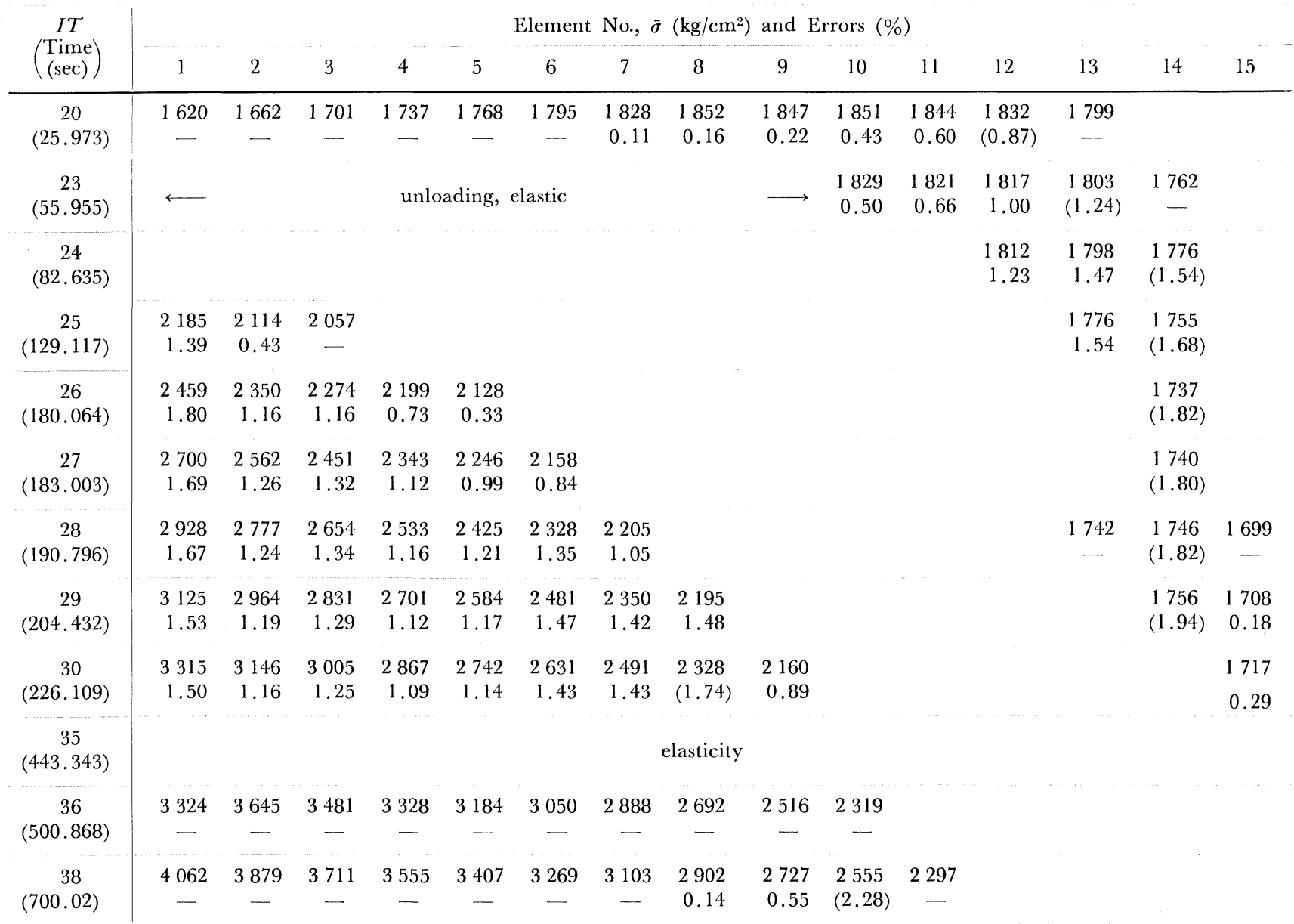

Note: $I T=$ number of thermal load increments.

$$
(\quad)=\text { max. error. }
$$

the mesh configuration, possibly less than $1 \%$ to the author's experience.

Accordingly, it can be concluded that the modified $r_{\text {min }}$ method II provides the most reliable solution of the three methods studied.

The computer program which is based on the Method III is capable of utilizing the iterative convergent algorithm by the modified Newton-Raphson method (i.e., constant stiffness matrix). However, the errors were only slightly improved with the adoption of the above algorithm. Just to be "safe", all the computations in the following section were done using the above algorithm. Furthermore, there are delicate problems associated with loading and unloading particularly for a cyclic thermal loading process, and without careful treatments of these problems a reliable solution cannot be obtained. These are omitted in this paper. The program also employs an automatic mesh generator with restarting and plotting capabilities to make analyses efficient.

\section{Application to Centrifugal Casting Mold}

1. Preparations for Computation

1. Transient Temperature Distribution

The method for calculating temperature distribu-
Error $=\frac{\bar{\sigma}-\bar{H}}{\bar{H}} \times 100(\%)$.

Upper figures denote $\bar{\sigma}$ and lower figures error.

tion of mold is described in a previous paper ${ }^{21)}$ and in the previous section. Therefore, only changes made in this section will be discussed here.

First, the heat transfer equation through refractory coating was revised and is given in Appendix $A$.

Second, the relationship between heat content $h$ and temperature $T$ above the solidus was determined by a thermal analysis experiment, as is seen in $A p$ pendix $B$.

In addition to the above-mentioned revisions, it is necessary to examine the validity of the treatment of refractory coating. For this purpose, it is most desirable to directly measure the temperature history of mold during casting. However, this is fairly difficult primarily because the mold rotates at considerably high speed. Therefore, in this work, instead of the direct measurement, $k$ and $\beta$ of Eq. (A-1) of Appendix $A$ were determined in such a way that the average cooling rates in the solidification range best fit the measured values of dendrite arm spacing (d.a.s.). For this purpose, the relationship between the average cooling rate and the d.a.s. obtained by Suzuki et al. ${ }^{24)}$ was used. Measured values of d.a.s. of HK40 centrifugal casting and casting conditions are available elsewhere. ${ }^{21)} \quad k$ and $\beta$ thus determined are $0.002\left(\mathrm{cal} / \mathrm{cm} \cdot \mathrm{sec} \cdot{ }^{\circ} \mathrm{C}\right)$ and 1.10 , respectively, 
which were used throughout in Chapter III.

2. Equivalent Strain Range and the Work due to Plastic Deformation

Various physical parameters have been proposed to evaluate the thermal fatigue behavior of metals. ${ }^{25}$ Among them, Taira et $a l .{ }^{26-28)}$ have used total equivalent strain range $\Delta \bar{\varepsilon}$ to estimate the thermal fatigue life under multiaxial thermal stresses and shown that the thermal fatigue life can be predicted using thermal fatigue or low cycle fatigue test data conducted under uniaxial stress condition. Accordingly, $\Delta \bar{\varepsilon}_{\max }$ at I.D., which is the maximum throughout the wall-thickness, was used as a parameter to estimate the thermal fatigue life or bore surface cracking.

Regarding the mold warping or bowing, it is known by experience that the severer the casting conditions, i.e., higher casting temperature and so on, the more rapid the warping or distortion rate and also that the thicker the mold wall, the more difficult for the mold to warp. Therefore, in this paper, to take into account these practical experiences the distortion rate is assumed to be described in terms of the work due to plastic deformation defined by

$$
\begin{aligned}
& W^{\mathrm{p}}=\sum_{i} d W_{i}^{\mathrm{p}} \\
& d W_{i}^{\mathrm{p}}=\frac{1}{V} \sum_{j=1}^{N} \bar{\sigma}_{i, j} d \bar{\varepsilon}_{i, j}^{\mathrm{p}} \operatorname{vol}_{j} \\
& V=\sum_{j=1}^{N} \operatorname{vol}_{j}
\end{aligned}
$$

where, vol: volume of element

$\mathrm{N}$ : number of elements

$W^{\text {": }}$ the "plastic work over a mold", thus depending not only on the extent of plastic deformation but also on the mold wall-thickness

Suffixes $i, j$ : thermal loading steps and element number, respectively.

$\Delta \bar{\varepsilon}_{\max }$ was taken as the value when the equivalent strain at the mold I.D. reaches the maximum in the $\bar{\sigma}-\bar{\varepsilon}$ hysteresis loop and $W^{\text {p }}$ was taken as the accumulated value by then.

\section{Computational Results and Discussion}

1. Influences of Pouring Temperature and the Thermal Resistance of Refractory Coating

Fixing the dimensions of the mold at $b_{2} / a_{2}=3.0$ and the preheating temperature of the mold at $250{ }^{\circ} \mathrm{C}$, a series of calculations were performed with the pouring temperature and the thermal resistance of coating changed. The $a$ and $b$ are the inner and outer radii and the suffixes 1 and 2 denote casting and mold, respectively. The wall-thickness of casting was fixed constant throughout this section at $a_{1} / b_{1}=0.655$. Dimensionless or normalized coating thicknesses $d / b_{1}$ were taken to be $0.007,0.014,0.028$ and 0.042 which correspond to the normalized thermal resistances of $3.51,7.05,14.19$ and $21.44\left(\mathrm{~cm} \cdot \mathrm{sec} \cdot{ }^{\circ} \mathrm{C} / \mathrm{cal}\right)$ as defined by

$$
\bar{H}_{R}=\frac{a_{2}}{k b_{1}} \ln \left(\frac{a_{2}}{b_{1}}\right)
$$

The results are shown in Figs. 6(a) to (c).

As anticipated, both $\Delta \bar{\varepsilon}_{\max }$ and $W^{\mathrm{p}}$ decrease as the pouring temperature decreases and as $\bar{H}_{R}$ increases, matching well with the maximum temperature $\mathcal{T}_{\max }$ at the mold I.D. For example, when $\bar{H}_{R}$ is increased from 5 to $10\left(\mathrm{~cm} \cdot \mathrm{sec} \cdot{ }^{\circ} \mathrm{C} / \mathrm{cal}\right)$ with the pouring temperature of $1580^{\circ} \mathrm{C}, T_{\max }$ decreases from 615 down to $535^{\circ} \mathrm{C}, \Delta \bar{\varepsilon}_{\max } 1.85$ down to $1.22 \%$ and $W^{\mathrm{p}} 1.5$ down to $0.96\left(\mathrm{~kg} / \mathrm{cm}^{2}\right)$. This effect is considerably

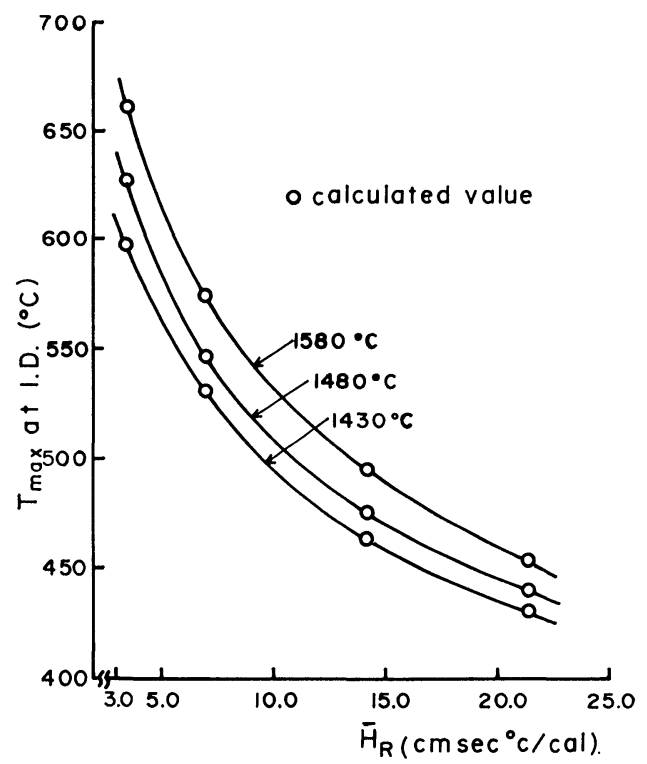

(a) The maximum temperature at mold I.D. $T_{\max }$,

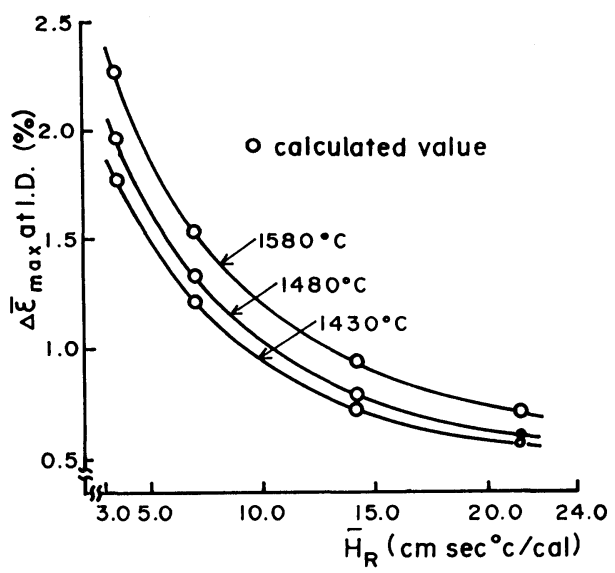

(b) The maximum equivalent strain range at mold I.D. $\Delta \bar{\varepsilon}_{\text {max }}$

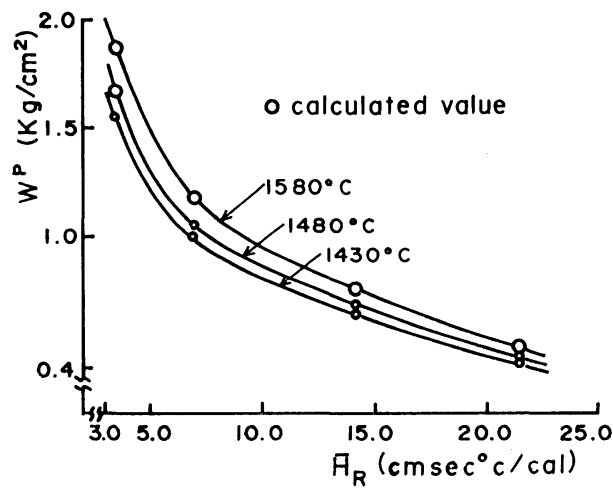

(c) The plastic work over a mold $W^{\mathrm{p}}$.

Fig. 6. The influences of pouring temperature and normalized thermal resistance of refractory coating $\bar{H}_{R}$. 
more significant to increase the thermal fatigue life of mold I.D. than decreasing the pouring temperature, e.g., from 1580 down to $1430^{\circ} \mathrm{C}$. Thus, the adiabatic effect of coating is found to be crucial.

\section{Influences of Mold Wall-thickness}

In order to examine the influences of the mold wall-thickness, the wall-thickness was varied from $b_{2} / a_{2}=2.0$ to 5.0 while fixing other conditions constant. The results are shown in Fig. 7. Throughout these four cases, the maximum temperatures at I.D. were essentially the same, i.e., $T_{\max }=545^{\circ} \mathrm{C}$. This is because when the temperature at I.D. reached the maximum, the temperature at O.D. was almost unchanged.

$W^{\mathrm{p}}$ decreased as was expected as the mold wallthickness increased. On the other hand, the increase of $\Delta \bar{\varepsilon}_{\max }$ over $b_{2} / a_{2}=3.0$ was fairly small and, contrary to expectation, there was no increase over $b_{2} / a_{2}=4.0$. This suggests that, when the mold warping is more critical than the bore surface cracking, relatively thicker walled-mold is desirable. This tendency of $W^{\mathrm{p}}$ and $\Delta \bar{\varepsilon}_{\max }$ is better interpreted by Fig. 8, in which the accumulated plastic work per unit volume was plotted vs. the normalized mold radius $r / b_{1}$. It is seen that the plastic zone spreads outward as the wall-thickness $\left(b_{2} / a_{2}\right)$ increases, but that it stops over $b_{2} / a_{2}=4.0$. Therefore, while the plastic zone remains unchanged (or $\Delta \bar{\varepsilon}_{\max }$ remains constant), $W^{\mathrm{p}}$ decreases as a result of increasing wallthickness. Thus, the trend of decreasing $W^{\mathrm{p}}$ with increasing mold wall-thickness is in accordance with already mentioned foundry experiences of mold warping or bowing.

\section{Influences of the Preheating Temperature of Mold}

Finally, the influences of the preheating temperature of mold were examined. The casting conditions are shown in Fig. 9 along with the results. $T_{\max }$ increases linearly with the preheating temperature. $\Delta \bar{\varepsilon}_{\max }$ also increases. Thus, the results indicate that it is desirable to keep the preheating temperature at a relatively low value.

\section{Summary}

(1) The modified $r_{\min }$ method II (Method III) proved to provide the most reliable solution among the three methods investigated. That is, the best followability of the equivalent stress and the flow stress of the material was obtained with exceedingly small relative errors between them. On the contrary, the iterative convergent method (Method I) adopting the effective stiffness matrix defined by Eq. (9) turned out to result in rather poor followability. The other modified $r_{\min }$ method (Method II) does not allow elements to yield one by one in the strict sense; hence, errors would be unavoidable and difficulties may arise particularly when complicated cyclic thermal loading is applied.

(2) A simple and efficient algorithm to determine time increment was presented. It was shown that when this algorithm is combined with the Method III, highly accurate solution can be obtained with considerably less thermal load increments in the light

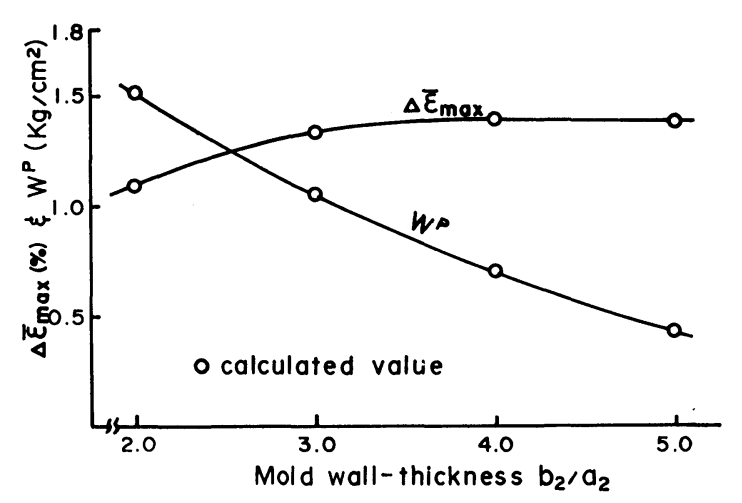

Casting wall-thickness: $a_{1} / b_{1}=0.655$

Normalized thermal resistance of coating $\bar{H}_{R}=7.05(\mathrm{~cm} \cdot \mathrm{sec}$. ${ }^{\circ} \mathrm{C} / \mathrm{cal}$ ),

Pouring temp.: $1480^{\circ} \mathrm{C}$

Preheating temp. of mold: $250^{\circ} \mathrm{C}$.

Fig. 7. The influences of mold wall-thickness $\left(b_{2} / a_{2}\right)$ on $\Delta \bar{\varepsilon}_{\max }$ and $W^{p}$.

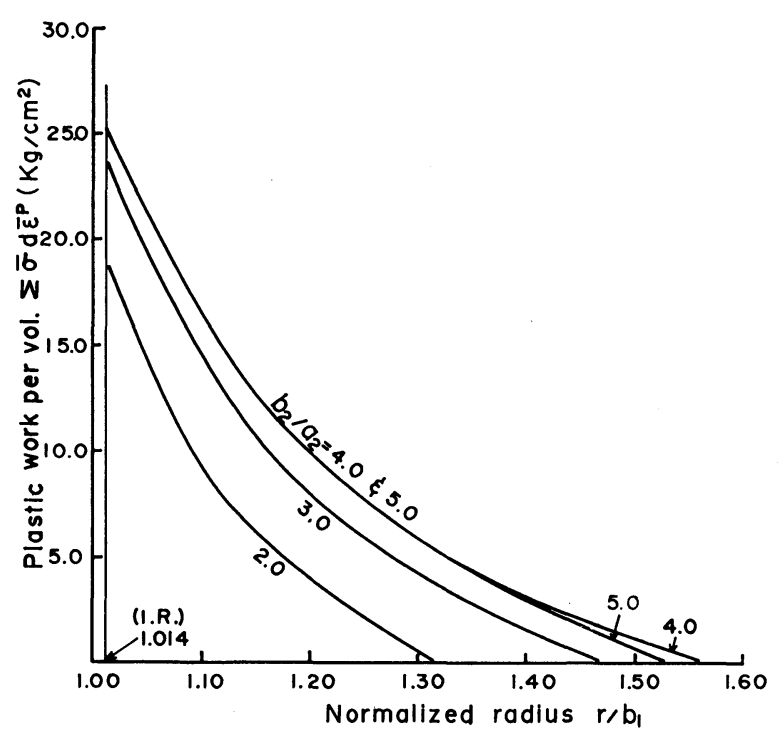

Fig. 8. The influence of mold wall-thickness on the distribution of the plastic work per volume $\sum_{i} \bar{\sigma}_{i} d \bar{\varepsilon}_{i}^{-\mathrm{p}}$ : Casting conditions are same as in Fig. 7.

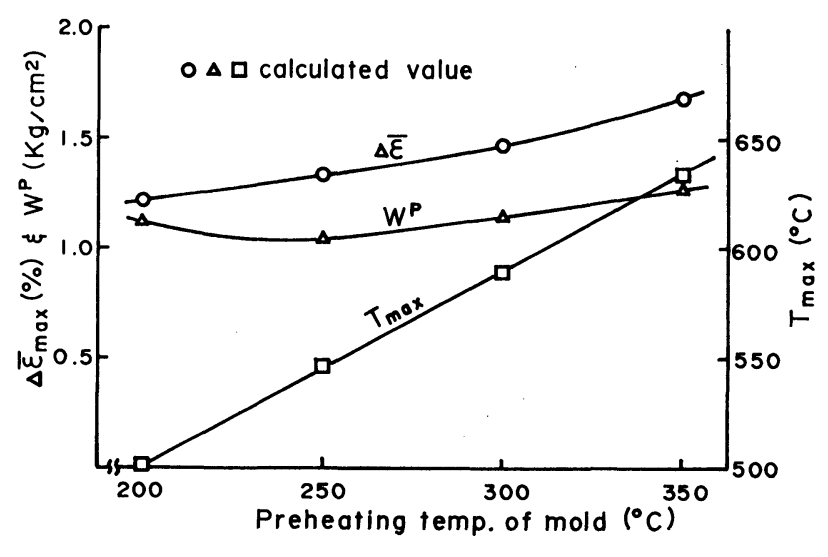

Casting and mold wall-thicknesses: $a_{1} / b_{1}=0.655$ and $b_{2} / a_{2}=3.0$, respectively,

Pouring temp.: $1480^{\circ} \mathrm{C}$

Normalized thermal resistance of coating $\bar{H}_{R}=$ $7.05\left(\mathrm{~cm} \cdot \mathrm{sec} \cdot{ }^{\circ} \mathrm{C} / \mathrm{cal}\right)$.

Fig. 9. The influences of the preheating temperature of mold on $T_{\max }, \Delta \bar{\varepsilon}_{\max }$ and $W^{p}$. 
of this kind of complicated thermal loading history.

(3) The influences of various casting parameters on the thermal fatigue of centrifugal casting mold were studied. Mold warping or distortion was also discussed in terms of $W^{\text {p }}$, "the plastic work over a mold " defined by Eq. (11). The results are shown in Figs. 6 to 9 .

\section{REFERENGES}

1) O. G. Zienkiewicz: The Finite Element Method in Engineering Science, McGraw-Hill Book Co., London, (1971).

2) C. S. Desai and J. F. Abel: Introduction to the Finite Element Method, Van Nostrand Reinhold, New York, (1972).

3) Y. Yamada: Plasticity and Visco-elasticity, Baifukan, Tokyo, (1972). (in Japanese)

4) Y. Yamada: Plasticity and Visco-elasticity, Baifukan, Tokyo, (1980). (in Japanese)

5) P. V. Marcal: Int. J. Mech. Sci., 7 (1965), 229.

6) P. V. Marcal and I. P. King: Int. J. Mech. Sci., 9 (1967), 143.

7) O. G. Zienkiewicz: Int. J. Num. Meth. Engineering, 1 (1969), 75.

8) O. G. Zienkiewicz and G. G. Nayak: Int. J. Num. Meth. Engineering, 5 (1972), 113.

9) F. G. Basombrío and G. S. Sarmiento: Nucl. Engineering. Des., 49 (1978), 231.

10) M. D. Snyder and K.J. Bathe: M.I.T. Rep. 82448-3, (1977).

11) Y. Yamada, N. Yoshimura and T. Sakurai: Int. J. Mech. Sci., 10, (1968), 343.

12) T. Inoue, K. Tanaka and M. Aoki: Trans. Japan Soc. Mech. Eng., 38 (1972), 2490.

13) T. R. Hsu and J.J.M. Too: Int. Conf. Pressure Vessel Technol. Vol. 3, No. 1, (1977), 83.

14) For example: J. Jezeirski: AFS Cast Metals Research J., (1970), June, 75

Y. Ebisu: unpublished paper (Feb. 1972)

15) For example: K. Yasue, M. Isotani, Y. Kondo and T. Nishio: Imono (J. Japan Foundrymen's Soc.), 54 (1982), 542.

16) R. Hill: The Mathematical Theory of Plasticity, Clarendon Press, Oxford, (1950).

17) F. A. McGlintock and A. S. Argon: Mechanical Behavior of Materials, Addison-Wesley Publ. Co., Mass., (1966), 283.

18) T. Inoue and K. Tanaka: Trans. Japan Soc. Mech. Eng., 22, (1973), 218. (in Japanese)

19) Y. Ueda and T. Yamakawa: Proc. Int'l. Conf. Mechanical Behavior of Materials, III, (1972), 10.

20) F. G. Rammerstorfor, D. F. Fischer, W. Mitter, K. J. Bathe and M. D. Snyder: Comp. Str., 13 (1981), 771.

21) Y. Ebisu: AFS Trans., 85 (1977), 643.

22) M. Sugiyama, T. Umeda, H. Takeuchi and H. Kato: Imono (J. Japan Fondrymen's Soc.), 47 (1975), 178.

23) Y. Nishida, M. Isotani and H. Matsubara: Imono (J. Japan Foundrymen's Soc.), 48 (1976), 299.

24) A. Suzuki, J. Nakamura and Y. Uchida: Imono ( $J$. Japan Foundrymen's Soc.), 40 (1968), 206.

25) For example: Thermal Stresses and Thermal Fatigue, ed. by S. Taira, Nikkan Kogyo Shimbun, Tokyo, (1974).

26) S. Taira, M. Ohnami, T. Inoue and F. Iori: J. Soc. Malerials Sci., Japan, 14 (1965), 988.

27) S. Taira, M. Ohnami, T. Inoue and F. Iori: J. Soc. Materials Sci., 16 (1967), 94.

28) S. Taira and T. Inoue: Proc. Int. Conf. on Thermal Stresses and Thermal Fatigue, Butterworths Sci. Publ., London, (1971), 5.

\section{Appendix $A$}

The revised heat transfer equation through refractory coating is

$$
\begin{aligned}
& w=w_{0} \exp \left(-\frac{\beta D}{b_{1}-a_{1}}\right) \\
& w_{0}=\left\{\begin{array}{ll}
\frac{k}{b_{1}} \ln \left(a_{2} / b_{1}\right) \\
\text { for casting/coating interface } \\
\frac{k}{a_{2}} \frac{\Delta T_{\mathrm{c}}}{\ln \left(a_{2} / b_{1}\right)} \\
\text { for } \mathrm{mold} / \text { coating interface }
\end{array}\right\}
\end{aligned}
$$

where, $w, w_{0}$ : heat flux from casting to mold (cal/ $\mathrm{cm}^{2} \cdot \mathrm{sec}$ )

$k$ : thermal conductivity of coating (cal/ $\mathrm{cm} \cdot \sec \cdot{ }^{\circ} \mathrm{C}$ )

$D: \quad$ solidified thickness of casting $(\mathrm{cm})$

$\Delta T_{\mathrm{c}}$ : temperature difference through coating thickness $\left({ }^{\circ} \mathrm{C}\right)$

$\beta$ : damping coefficient to retard heat flow rate (-)

$a_{1}, b_{1}$ : inner and outer radii of casting $(\mathrm{cm})$ $a_{2}, b_{2}$ : inner and outer radii of mold $(\mathrm{cm})$.

The above equation takes account for the curvature effect of mold, and it is considered reasonable in the light that the thermal resistance at the metal/mold interface increases roughly linearly with coating thickness in the metal mold casting experiments by Sugiyama et al. ${ }^{22)}$ and Nishida et al. ${ }^{23)}$

\section{Appendix B}

\section{h-T Relation above the Solidus Temperature}

$5 \mathrm{~kg}$ of HK40 alloy was induction-melted and the time-temperature curve during solidification was obtained by a thermo couple. The heat content $h$ $v s$. temperature $T$ relation was determined by melting the same amount of reference metal or pure nickel whose specific heat is known. The specific heat gives the heat extraction rate from the melt. The $h-T$ relations thus obtained were properly linearized as follows :

For solidification range,

$$
\begin{gathered}
h=0.194 T-61.55(\mathrm{cal} / \mathrm{g}) \\
\left(1300^{\circ} \mathrm{C} \leqq T \leqq 1350^{\circ} \mathrm{C}\right) \\
h=0.41 T-353.15(\mathrm{cal} / \mathrm{g}) \\
\left(1350^{\circ} \mathrm{C} \leqq T \leqq 1380^{\circ} \mathrm{C}\right) \\
h=1.20 T-1443.35(\mathrm{cal} / \mathrm{g}) \\
\left(1380^{\circ} \mathrm{C} \leqq T \leqq 1395^{\circ} \mathrm{C}\right) \\
h=8.2 T-11208.35(\mathrm{cal} / \mathrm{g}) \\
\left(1395^{\circ} \mathrm{C} \leqq T \leqq 1400^{\circ} \mathrm{C}\right)
\end{gathered}
$$

Above the liquidus,

$$
h=0.175 T+26.65(\mathrm{cal} / \mathrm{g})\left(T \geqq 1400^{\circ} \mathrm{C}\right)
$$

